\title{
Microwave Crosstalk in Lumped Element Far-IR MKIDs
}

\author{
Omid Noroozian $^{\mathrm{a}}$, Peter K. Day ${ }^{\mathrm{b}}$, Byeong Ho Eom ${ }^{\mathrm{a}}$, Henry G. LeDuc ${ }^{\mathrm{b}}$, Jonas Zmuidzinas ${ }^{\mathrm{a}}$ \\ ${ }^{a}$ California Institute of Technology, Pasadena, CA 91125, USA \\ ${ }^{\mathrm{b}}$ Jet Propulsion Laboratory, Pasadena, CA 91109, USA
}

\begin{abstract}
We have made close-packed far-infrared MKID arrays with $\sim 250$ pixels using TiN on silicon. Measurements show a large scatter in quality factor arising from crosstalk. This is confirmed by pump-probe experiments and EM simulations. Our new shielded resonator designs show very low crosstalk levels.
\end{abstract}

\section{INTRODUCTION AND BACKGROUND}

A $\mathrm{N}$ MKID is a photon detector that uses the change in surface impedance of a superconducting resonator to detect light. The resonator is weakly coupled to a CPW or CPS feed line. The amplitude and phase of a microwave probe signal transmitted on the feed line past the resonator changes as photons break cooper pairs. Hundreds to thousands of resonators tuned to slightly different frequencies can be coupled to a single feed line. In order to achieve next generation large scale arrays with $\sim 1000$ resonators per microwave coax, high multiplexing factors and packing densities are needed. This however, can lead to crosstalk between the microwave resonators and requires careful design considerations to overcome.

\section{RESULTS}

We have fabricated close-packed lumped element MKID $^{1}$ arrays (Fig. 1) with $\sim 250$ pixels for the far-infrared $350 \mu \mathrm{m}$ band using a single layer of $\mathrm{TiN}^{2}$ on high resistivity silicon substrates. Our layers are $20-40 \mathrm{~nm}$ thick with Tc ranging between 1-4.5 K and normal-state resistivity of $\rho_{\mathrm{n}} \sim 100$ $\mu \Omega \mathrm{cm}$. These resonators have meandered or spiral inductors and interdigitated capacitors (IDC) and have frequencies of $2 \mathrm{GHz}$ separated by $\sim 1 \mathrm{MHz}$ in frequency and $\sim 65 \mu \mathrm{m}$ in space. The resonators were designed for a constant coupling quality factor (Qc) but show a large scatter (2-4 orders of magnitude) in $\mathrm{Q}_{c}$ which is almost certainly due to multiresonator modes arising from coupling between pixels. Our electromagnetic simulations predict interpixel coupling (Fig. 2) and also reproduce the observed distribution of mode frequencies. In addition, microwave pump-probe experiments directly reveal crosstalk as large as $\sim 70 \%$ for our first design.

We have recently designed and measured new resonators showing very low crosstalk levels. We will present preliminary results including experimental data, electromagnetic simulations, and a simple circuit model predicting the crosstalk.

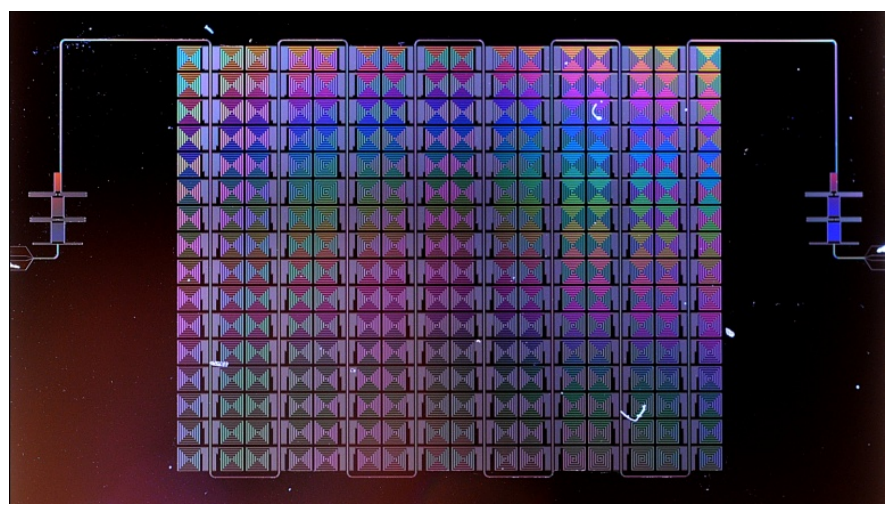

Figure 1. A 16x16 array of far-infrared pixels with a meandered feed line and two on-chip transformers.

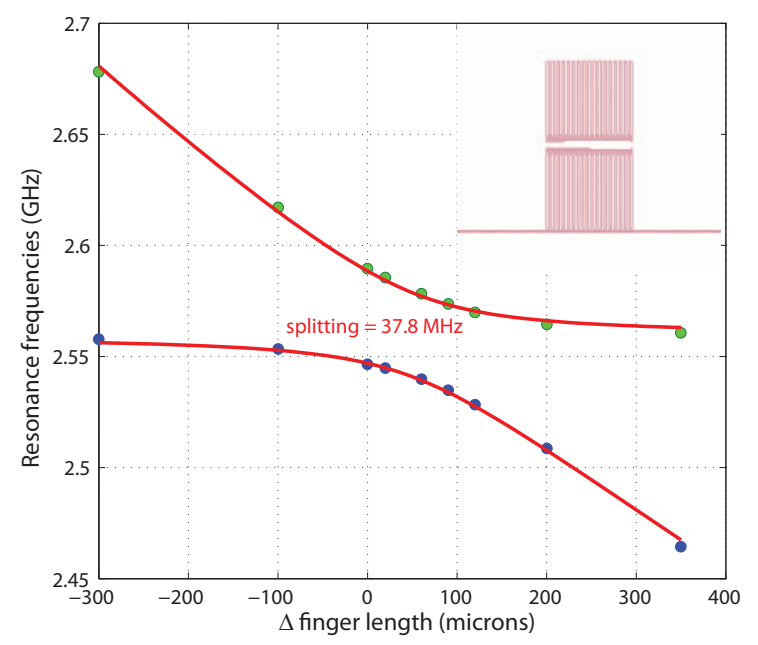

Figure 2. The resonance frequencies of two coupled resonators (inset) when the finger length of one capacitor is changed show an avoided level crossing indicating a cross-coupling strength of $37.8 \mathrm{MHz}$.

\section{REFERENCES}

[1] S. Doyle, P. Mauskopf, J. Naylon, A. Porch, and C. Duncombe, "Lumped Element Kinetic Inductance Detectors," Journal of Low Temperature Physics, vol. 151, pp. 530-536, April 2008.

[2] H. G. Leduc, B. Bumble, P. K. Day, A. D. Turner, B. H. Eom, S. Golwala, D. C. Moore, O. Noroozian, J. Zmuidzinas, J. Gao, B. A. Mazin, S. McHugh, and A. Merrill, "Titanium Nitride Films for Ultrasensitive Microresonator Detectors," submitted to Appl. Phys. Lett., 29 March 2010, [online]. Available: arXiv:1003.5584v1. 